\title{
Editorial1 - Die Öffentlichen Finanzen im Umbruch
}

von Uwe Wagschal

Der Zustand der öffentlichen Finanzen scheint aktuell auf einem guten Weg zu sein. Zum wiederholten Male schreibt 2014 der öffentliche Gesamthaushalt Überschüsse und auch der Bundeshaushalt ist zum ersten Mal seit über 40 Jahren im Plus. Rekordsteuereinnahmen und eine gute Lage bei den Sozialversicherungen lassen den Zustand der öffentlichen Finanzen so positiv wie seit langem nicht erscheinen. Ab 2016 soll dann, so die Neuregelung des Art. 115 (2) GG, der Haushalt dauerhaft für den Bund ausgeglichen sein (wobei ein bescheidenes 0,35\% des BIP zulässig sein soll). Nur in Ausnahmefällen soll ein Defizit zugelassen sein, welches jedoch wieder über ein Kontrollkonto ausgeglichen werden muss. Für die Länder, so die Regelung des Art. 109 (3) GG gilt dann ab 2020, dass überhaupt keine Schulden mehr aufgenommen werden dürfen. In Zukunft würde dies bedeuten, dass die Verschuldung deutlich heruntergefahren wird und die Schuldenquoten, bei einem langfristig angenommen Wachstum des BIP von 1,5\% und einer Inflation von $2 \%$, deutlich sinken würden. Eine Rückführung der Schuldenquote innerhalb der nächsten zehn Jahre unter dem Maastricht Grenzwert von $60 \%$ des BIP (2013 rund 78,4 \% des BIP) wäre damit realistisch und möglich.

Weitere Schuldengrenzen, die für die Bundesrepublik gelten, gehen auf supranationale Vereinbarungen zurück, wie die Drei-Prozent-Hürde des Vertrages von Maastricht für die gesamtstaatlichen Haushaltsdefizite sowie die 2012 im Fiskalvertrag vereinbarten, wesentlich strengeren $0,5 \%$ des zulässigen strukturellen Defizits des Haushaltes. Vor dem Hintergrund des seit 1970 fast kontinuierlich ansteigenden Verschuldungsniveaus kann dabei mit Fug und Recht von einem

1 Anmerkung der Schriftleitung: Aufgrund eines technischen Versehens wurde für das Heft 1/2014 der ZSE eine unkorrigierte Fassung der Seite 1 übernommen. Wir bitten das zu entschuldigen. 
Umbruch der öffentlichen Finanzen gesprochen werden, sollten die Regeln tatsächlich eingehalten werden. Hier taucht jedoch ein erstes Fragezeichen auf: ${ }^{2}$ Denn in der Summe sind die Regelverletzungen der europäischen Schuldenregeln häufiger als die Regelbefolgungen.

Der Wandel der öffentlichen Finanzen dokumentiert sich an mindestens vier bemerkenswerten Umbrüchen:

1. den Veränderungen in der öffentlichen Haushaltswirtschaft im Gefolge der Wirtschafts- und Finanzkrise

2. der Entwicklung hin zu ausgeglichenen Haushalten in Deutschland

3. die sich abzeichnenden Veränderungen im Hinblick auf den zukünftigen Finanzausgleich des Solidarpaktes und der des Solidaritätszuschlages

4. die Rückkehr des Keynesianismus als Herausforderung zur Legitimierung gesunder Finanzen

\section{Veränderungen im Gefolge der Wirtschafts- und Finanzkrise}

Die ersten Jahre nach der Lehman-Pleite 2008 waren gekennzeichnet von starken expansiven Reaktionen auf die einbrechenden Wirtschaftswachstums-Zahlen und die Schieflage der Banken und einzelner Länder während der Finanzkrise. Staaten, insbesondere Länder wie die USA, Australien, Korea reagierten mit erhöhten Ausgabenprogrammen und Steuerkürzungen. Auch die Bundesrepublik Deutschland hat solche Programme in diesen Jahren aufgelegt, jedoch wurden diese zügig beendet und die Ausgaben wurden ab 2010 wieder deutlicher konsolidiert. Ein Überblick über den Konsolidierungsfortschritt innerhalb der OECD-Welt gibt nachfolgende Abbildung 1, die die Konsolidierungsanstrengungen anhand eines zehnstufigen Indikators deutlich macht. ${ }^{3}$ So sind im Zeitverlauf schon die Spuren der Finanzkrise bei den Konsolidierungsbemühungen der betrachteten OECD Länder eindeutig sichtbar. Man sieht in Abbildung 1 deutlich, wie die Konsolidie-

2 Wagschal, U: Allheilmittel oder Budgetmimikry: Wie wirksam sind Verschuldungsgrenzen zur Haushaltskonsolidierung?, in: Zeitschrift für Zeitschrift für Staats- und Europawissenschaften, 3 (2011), 352 382 .

3 Insgesamt umfasst der Konsolidierungsindikator 10 Ausprägungen (Punkte): Keine Konsolidierung wird operationalisiert über einen (im Durchschnitt der Untersuchungsperiode) negativen Primärsaldo sowie einen Anstieg/Rückgang (1/2 Punkte) der Staatsverschuldungsquote im Untersuchungszeitraum. Eine teilweise Konsolidierung liegt vor, wenn der Primärsaldo im Durchschnitt zwischen 0 und $2 \%$ des BIP beträgt. Dabei werden vier Subtypen unterschieden (je nach Entwicklung der Schuldenquote im Untersuchungszeitraum sowie in den folgenden drei Jahren, 3-6 Punkte). Eine substanzielle Konsolidierung liegt vor, wenn der Primärsaldo im Durchschnitt der Untersuchungsperiode mindestens 2,0 \%des BIP beträgt. Dabei werden vier Subtypen unterschieden (je nach Entwicklung der Schuldenquote im Untersuchungszeitraum sowie in den folgenden drei Jahren, 7-10 Punkte). 
rungsanstrengungen (gemessen am OECD-Durchschnitt) mit dem Konjunkturverlauf zusammenhängen. Zum Zeitpunkt der ersten Ölpreiskrise (1980-1982), während der Krise Anfang der 1990er Jahre (1992-1995), der Finanzkrise (2007-2010) und nach dem Platzen der der Internet-Blase und den Terroranschlägen 2001 wurde dementsprechend auch der Rückgang der Konsolidierungsperformanz sichtbar. Die größten Konsolidierungsanstrengungen während der letzten 34 Jahre waren um die Millenniumswende zu beobachten. Hier konsolidierten vorwiegend europäische Länder besonders stark, um die Eintrittskriterien für den Eurowährungsraum zu erfüllen. Deutschland war nach dem Machtwechsel von 1982 besonders aktiv sowie seit 2007. In beiden Fällen liegt die Bundesrepublik oberhalb des Konsolidierungsdurchschnitts.

Abbildung 1: Konsolidierung 1980-2013 (3-jährige gleitende Durchschnitte)

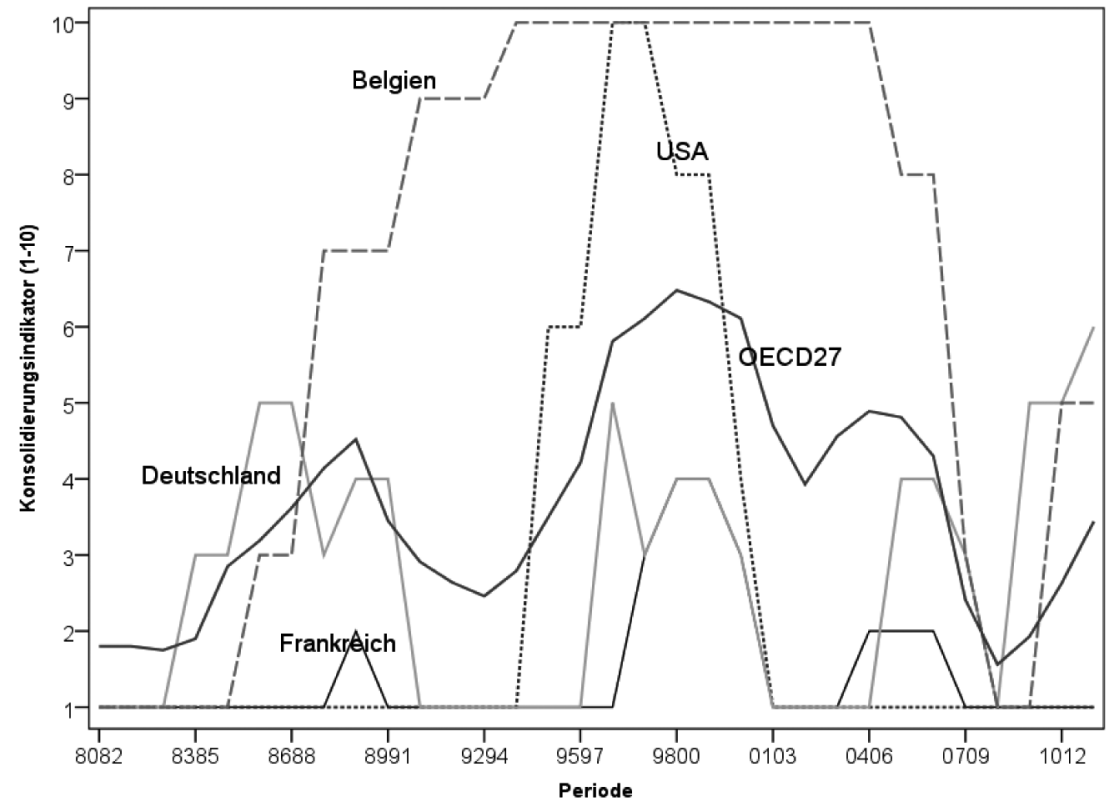

Anmerkungen: 27 OECD-Länder im Vergleich, eigene Auswertung auf Basis von OECDDaten für den im Text beschriebenen Konsolidierungsindikator

Allerdings muss man im Fall von Deutschland auch festhalten, dass die Finanzierung der deutschen Einheit als historische Ausnahmesituation - zu Recht - zu einem Gutteil über Kreditaufnahme geschah, so dass der Konsolidierungsindikator 
sich im Zeitraum ab 1991 bis 1995 auf dem niedrigsten Niveau bewegte. Im langfristigen Vergleich war Frankreich insgesamt das schlechteste Land im Konsolidierungsbenchmarking, während Belgien am besten abschnitt.

\section{Ausgeglichene Haushalte in Deutschland}

Die Entwicklung der öffentlichen Haushalte war im Aggregat in den vergangenen drei Jahren so positiv wie seit über 40 Jahren nicht. Nach der Verarbeitung der Weltwirtschaftskrise gelang es Deutschland, schneller als anderswo, die Haushalte auf einen Konsolidierungspfad zurückzuführen (vgl. auch Abb. 1). Dabei zeigt sich deutlich, dass Deutschland eine durchaus beachtliche Konsolidierungsleistung erbracht hat. Diese basiert in den letzten Jahren allerdings auch auf guten Rahmenbedingungen, wie den rückläufigen Zinsen, die allein für den Bund pro Jahr ca. 20 Mrd. Einsparungen hervorbringen, einer florierenden Exportwirtschaft sowie einer guten Lage auf dem Arbeitsmarkt, die es ermöglicht hat die Sozialkassen zu Konsolidierungszwecken einzusetzen.

Als zentrales Instrument hierbei gilt die 2009 eingeführte Schuldenbremse, die zumindest als Druckmittel für die Haushaltskonsolidierung positiv wirkt. Ebenso bedeutend ist aber auch die Umstellung des Budgetprozesses auf ein Top-DownVerfahren. Vor dem aktuellen Hintergrund einer sich eintrübenden Konjunktur, wieder aufscheinender Probleme in einigen Euroländern sowie eines deutlichen Rückgangs der Konsumzufriedenheit und des Unternehmensvertrauens (Rückgang des Ifo-Index, drastische Reduktion der Erwartungen zum Wirtschaftswachstum) bleibt abzuwarten, ob die Schuldenbremse tatsächlich langfristig die Verschuldung eindämmen kann. Ungeachtet des Anstiegs der Verschuldung waren die parallel eingeführten Schuldendefizitausgaben und Ausgabengrenzen auf europäischer Ebene jedoch nicht in der Lage die Verschuldung in den vergangenen Jahren einzudämmen.

Die Einstellungen gegenüber Staatsverschuldung sind dagegen - konstant durch Umfragen gemessen - gesamtgesellschaftlich negativ. Insofern erreicht die Politik an dieser Stelle auch die öffentliche Unterstützung, die für die Durchführung einer solchen Politik notwendig ist. 


\section{Neuer Finanzausgleich und Auslaufen des Solidarpaktes}

Der Koalitionsvertrag der Bundesregierung ${ }^{4}$ sieht vor, eine dritte Föderalismuskommission einzurichten. Es ist offensichtlich, dass einige strittige Punkte nicht im Koalitionsvertrag Erwähnung fanden und auch nicht alleine durch den Bund entschieden werden können. So sind bei verschiedenen anstehenden Problemen wie der Reform des Länderfinanzausgleichs, der Einhaltung der Schuldenbremse, das Auslaufen des Solidarpaktes und der Zukunft des Solidaritätszuschlags neben dem Bund auch Länder und die kommunale Ebene betroffen. Der Zeitplan dieser Verhandlungen, allgemein auch als Föderalismuskommission Föko III bezeichnet, sieht vor bis Ende 2015 eine Einigung zu finden, welche bis Ende 2016 in ein Gesetz gegossen werden soll. Insgesamt geht es dabei um ein Umverteilungsvolumen von ca. 30 Milliarden Euro.

Das Bundesfinanzministerium wies dabei Forderungen der Länder nach bis zu 30 Milliarden Euro zurück und verwies auf notwendige Strukturveränderungen, um Länderfinanzen dauerhaft auf eine solide Basis zu stellen. Insbesondere die Verteilung des Solidaritätszuschlags (15 Milliarden Euro jährlich an Einnahmen für den Bund, Tendenz steigend) ist dabei umstritten. So möchten die Länder mindestens die Hälfte, wenn nicht sogar alles vom Soli-Zuschlag, was den Bund aber enorm belasten würde. Zahlreiche weitere Vorschläge sind dabei auf dem politischen Markt. So möchten die Länder den „Soli“ entweder als eine Ergänzungsabgabe erhalten oder ihn in die Gemeinschaftssteuern integrieren. Dabei wird allerdings gerne die Genese dieser Zuschlagssteuer vergessen. So hatten 1993 die Länder im Rahmen der Soli-Einführung einen höheren Anteil an der Umsatzsteuer erhalten (sieben Punkte), die der Bund bei einer Länderbeteiligung am Soli nun nicht mehr leisten will. Die Länder möchten überdies, dass der Bund weitere Sozialausgaben übernimmt (wie etwa bei der Grundsicherung im Alter und dem BAFöG), ihnen bei den Zinslasten der Altschulden und bei der Sanierung der kommunalen Infrastruktur hilft. So verwundert es nicht, dass die Vorschläge zur Solidaritätsschlag auf einem Kontinuum zwischen Abschaffung (z.B. Söder) bis hin zur Beibehaltung (Walter-Borjans) liegen. Einige Stimmen (z.B. Nils Schmid) fordern die Kopplung an ein Arrangement zum Länderfinanzausgleich und der Lösung der Altschuldenproblematik der Länder. Der niedersächsische Ministerpräsident Weil setzt sich für die Beibehaltung des „Solis“ ein, wobei die Nutzung

4 Vgl. Koalitionsvertrag zwischen CDU, CSU und SPD: Deutschlands Zukunft gestalten, 18. Legislaturperiode, 2013. 
der Mittel für einen Fonds zur Tilgung von Altschulden eingesetzt werden könnten.

Ein bemerkenswerter, bereits schon früher einmal beerdigter Vorschlag, der jedoch den Föderalismus wieder beleben würde, findet Wiederauferstehung. So hat Bundesfinanzminister Schäuble die Forderung nach mehr Steuerautonomie erhoben. Mehr Steuerautonomie wurde schon in früheren Gesprächen über Gemeindefinanzreform vorgeschlagen und auch schon zu Zeiten der Föderalismuskommission I vom damaligen Ministerpräsidenten Bayerns Stoiber ins Spiel gebracht. Dabei werden $\mathrm{Zu}$ - und Abschlagsrechte der Länder auf die Einkommenssteuer von bis zu drei Prozentpunkten diskutiert. Allerdings wurde und wird diese Flexibilisierung von der Länder-Mehrheit abgelehnt.

Die SPD argumentiert, dass Steuerdumping aufgrund regional verschiedener Steuersätze droht sowie Steuerschlupflöcher und Steuerflucht möglich werden, obwohl diese Schlupflöcher europaweit geschlossen werden sollen. Eine Ausnahme in der SPD-Ablehnungsphalanx ist der Finanzminister von Baden-Württemberg Nils Schmid, der durchaus mehr Steuerautonomie der Länder befürwortet.

Auch im Hinblick auf den Finanzausgleich sind verschiedenste Positionen erkennbar. Bayern, welches neben Hessen, aktuell sogar gegen den geltenden Finanzausgleich klagt, prescht dabei am weitesten nach vorne. Der bayerische Finanzminister Söder legte einen 10-Punkte-Plan vor, der u.a. folgende Forderungen enthielt:

- $\quad$ eine strengere Finanzaufsicht über verschuldete Länder; Belohnung für Fortschritte, Bestrafung für Verschuldung;

- massive Kürzung des Länderfinanzausgleichs (Kürzung der bayerischen Zahlungen um 1 Mrd Euro pro Jahr;

- Begrenzung der bayerischen Zahlungen in den Finanzausgleich auf max. 1 Mrd. Euro pro Jahr;

- $\quad$ Einfrieren der Zahlungen in den Ausgleichstopf bis 2019;

- Zuschlag für die Stadtstaaten Berlin, Bremen und Hamburg soll abgeschafft werden;

- der Bund soll einen Teil der Bundesaufgaben von Berlin übernehmen.

Ein aktueller Vorschlag aus dem Finanzministerium birgt weiteren Zündstoff. So hat sich Bundesfinanzminister Schäuble nach den Klagen der Länder dazu bereit erklärt die Schuldenbremse für die Länder unter bestimmten Bedingungen zu lockern. Obwohl die Regel für die Bundesländer noch nicht gilt, soll sie also schon im Vorfeld wieder geändert werden. Der Vorschlag sieht so aus, dass von den $0,35 \%$ des zulässigen Defizits Deutschlands, sich der Bund nur bis zu 0,2 \% neu 
verschulden darf, während die Länder dafür über 0,15 \% davon „konsumieren“ dürfen. Dies soll allerdings an harte Bedingungen geknüpft werden. So soll der Stabilitätsrat in eine echte Kontrollbehörde umgewandelt werden, die Sanktionen aussprechen kann. Überdies sollen wohl auch Änderungen der Abstimmungsregeln im Stabilitätsrat für Sanktionen vorgenommen werden: Einfache Mehrheit statt Zweidrittelmehrheit.

\section{Die Rückkehr des Keynesianismus}

Die Rückkehr des Keynesianismus als Herausforderung für eine solide Stabilitätsund Haushaltsführung manifestiert sich in verschiedener Weise. Aktuell fordert etwa der IWF Deutschland und andere Länder auf mehr Geld für die Konjunkturbelebung auszugeben. Das alte „Lokomotivenargument“ und die Argumentationsfigur ,aus der Krise herauswachsen“ feiern eine Wiederauferstehen. Dabei wird aber empirisch übersehen, dass in den vergangenen Jahrzehnten die Verschuldung im Trend anwuchs, die Investitionen jedoch zurückgingen. Die Kritik an einer sparsamen Haushaltsführung war in der Literatur immer präsent gewesen. ${ }^{5}$ Auch angesichts der hohen Arbeitslosigkeit in zahlreichen Eurostaaten wird die Politik einer sparsamen Haushaltsführung jedoch wieder vermehrt kritisiert. ${ }^{6}$ Die Ernennung des französischen Finanzministers Pierre Moscovicis zum Kommissar für Wirtschafts- und Finanzangelegenheiten, Steuern und Zoll passt in dieses Bild. Nach Griechenland, Italien und Portugal ist Frankreich dasjenige Land welches das $3 \%$-Defizitkriterium des Vertrages von Maastricht am häufigsten in Europa gebrochen hat. Und mit Moscovici kommt nun jemand in dieses zentrale Amt, der besonders lax beim Schuldenabbau und dem Einhalten der europäischen Regeln agiert hat. Die in den letzten Jahren beschlossenen Verschärfungen des europäischen Stabilitätspaktes dürften damit ihr Drohpotenzial für die Haushaltssünder verlieren. Die im September 2014 beschlossenen Verlängerungen des Schuldenabbaupfades für Frankreich durch den ECOFIN dokumentieren dies bereits jetzt eindrucksvoll.

Die Diagnose, dass eine harte Austeritätspolitik für Verwerfungen auf den Arbeitsmärkten mit ursächlich ist, kann empirisch mehr als in Frage gestellt werden.

5 Vgl. Krugman, P. R.: The return of depression economics and the crisis of 2008, New York u.a., 2009.;. Flassbeck, H.: Zehn Mythen der Krise, Frankfurt, 2012.

6 Vgl. Schui, F.: Austerität: Politik der Sparsamkeit: Die kurze Geschichte eines großen Fehlers, München, 2014 . 
Die Kausalität kann bezweifelt werden. ${ }^{7}$ Diese Kritik unterliegt zudem einem gewissen Fehlschluss. Denn die vergangenen 50 Jahre Wirtschaftspolitik in Europa folgten zumeist genau dieser Logik, die eine immer höhere Verschuldung produzierte. Auf der anderen Seite wurde die höhere Verschuldung jedoch nicht für mehr Investitionen eingesetzt, sondern für einen deutlichen Anstieg der Sozialausgaben verwendet. Eine detaillierte Betrachtung des Verhältnisses der Sozialausgaben und der öffentlichen Investitionen über die vergangenen 40 Jahre zeigt, dass hier ein eher negativer Zusammenhang besteht und die Verschuldung nicht für dieses vermeintliche beschäftigungssteigernde Projekt eingesetzt wurde.

Neben diesen vier langfristigen Trends und Veränderungen kann die Frage gestellt werden, ob neben der Schuldenbekämpfung und Haushaltskonsolidierung auch in den anderen Bereichen der Staatsfinanzen, also der Steuerpolitik und den Staatsausgaben, eher kurz- und mittelfristige Umbrüche in Deutschland zu identifizieren sind.

\section{Steuerpolitische Entscheidungen der Bundesregierung}

Verglichen mit dem großen Versuch des Umsteuerns bei der Verschuldung und den Haushaltsdefiziten, sind die steuerpolitischen Entscheidungen der Bundesregierung für die aktuelle Legislaturperiode eher unbedeutend. Die steuerpolitischen Themen sind im Koalitionsvertrag von 2013 deutlich weniger prominent vertreten als etwa in den letzten drei Koalitionsverträgen zuvor. ${ }^{8}$ Die Übersicht in Tabelle 1 fasst die wichtigsten Aussagen des Koalitionsvertrags zu den einzelnen steuerpolitischen Themen zusammen. Dabei kann man auf Basis eines Vergleichs mit der steuerpolitischen Programmatik in den Wahlprogrammen auch ablesen, welche Partei sich bei welchem Thema überwiegend durchgesetzt hat. So ist der Verzicht auf generelle Steuererhöhungen ein deutlicher Sieg für die Unionsparteien, wenn gleich diese nicht völlig ausgeschlossen werden. Explizit wird eine Erhöhung der Erbschaftssteuer ausgeschlossen. Insgesamt wird bei der Betrachtung von Tabelle 1 deutlich, dass sich die CDU/CSU bei den steuerpolitischen Themen eher durchsetzen konnte als die SPD. Die größten Erfolge der SPD liegen beim Verzicht auf eine Milderung der kalten Progression sowie bei der Senkung der Stromsteuer.

7 Vgl. Reinhart, C. M. /Rogoff, K. S.: This Time Is Different: A Panoramic View of Eight Centuries of Financial Crises, Princeton, 2009.

8 Vgl. Wagschal, U.: Der Koalitionsvertrag und seine steuerpolitischen Inhalte, in: Institut für Finanzen und Steuern (IFST): Die Steuerpolitik der neuen Legislaturperiode, IFST-Schrift 496 (2014), 7-28. 
Tabelle 1: Steuerentscheidungen im Koalitionsvertrag

\begin{tabular}{|c|c|c|}
\hline Thema & Aussage im Koalitionsvertrag & $\begin{array}{l}\text { Welche Partei } \\
\text { hat sich über- } \\
\text { wiegend durch- } \\
\text { gesetzt? }\end{array}$ \\
\hline $\begin{array}{l}\text { Steuererhö- } \\
\text { hungen }\end{array}$ & $\begin{array}{l}\text { Grundsätzlich soll auf Steuererhöhungen verzichtet wer- } \\
\text { den, jedoch werden sie nicht explizit ausgeschlossen (Ko- } \\
\text { alitionsvertrag: S. 89). }\end{array}$ & $\mathrm{CDU} / \mathrm{CSU}$ \\
\hline $\begin{array}{l}\text { Vermögens- } \\
\text { und Erb- } \\
\text { schafts- } \\
\text { steuer }\end{array}$ & $\begin{array}{l}\text { Keine Erhöhung der Erbschaftssteuer: „Unternehmens- } \\
\text { nachfolge soll auch künftig durch die Erbschaftsbesteue- } \\
\text { rung nicht gefährdet werden.“ (Koalitionsvertrag: S. 35) } \\
\text { Keine Aussagen zur Vermögenssteuer }\end{array}$ & $\mathrm{CDU} / \mathrm{CSU}$ \\
\hline $\begin{array}{l}\text { Kalte Pro- } \\
\text { gression }\end{array}$ & $\begin{array}{l}\text { Keine Aussagen im Koalitionsprogramm. Die SPD hat } \\
\text { eine Senkung der kalten Progression bereits } 2012 \text { im Bun- } \\
\text { desrat verhindert. }\end{array}$ & SPD \\
\hline $\begin{array}{l}\text { Ehegatten- } \\
\text { splitting }\end{array}$ & $\begin{array}{l}\text { „Wir wollen die Akzeptanz des Faktorverfahrens für Ehe- } \\
\text { gatten stärken.“( (SPD-Vorhaben) }\end{array}$ & $\begin{array}{l}\text { CDU/CSU (es } \\
\text { bleibt beim Sta- } \\
\text { tus Quo) }\end{array}$ \\
\hline $\begin{array}{l}\text { Kapitalver- } \\
\text { mögen }\end{array}$ & $\begin{array}{l}\text { Keine Aussage zur Abgeltungssteuer oder anderer Besteu- } \\
\text { erung des Kapitalvermögens }\end{array}$ & $\mathrm{CDU} / \mathrm{CSU}$ \\
\hline $\begin{array}{l}\text { Finanztrans- } \\
\text { aktions- } \\
\text { steuer }\end{array}$ & $\begin{array}{l}\text { „Wir wollen eine Finanztransaktionssteuer mit breiter Be- } \\
\text { messungsgrundlage und niedrigem Steuersatz zügig um- } \\
\text { setzen und zwar im Rahmen einer verstärkten Zusammen- } \\
\text { arbeit in der EU.“ (Koalitionsvertrag: S. 64) }\end{array}$ & $\begin{array}{l}\text { Position wird } \\
\text { von allen Koali- } \\
\text { tionsparteien } \\
\text { vertreten }\end{array}$ \\
\hline $\begin{array}{l}\text { Gewerbe- } \\
\text { steuer }\end{array}$ & $\begin{array}{l}\text { „Die Gewerbesteuer ist eine wichtige steuerliche Einnah- } \\
\text { mequelle der Kommunen. Wir wollen, dass auf der Basis } \\
\text { des geltenden Rechts für die kommenden Jahre Planungs- } \\
\text { sicherheit besteht“ (S. 94). }\end{array}$ & $\begin{array}{l}\text { CDU/CSU (Sta- } \\
\text { tus Quo-Orien- } \\
\text { tierung) }\end{array}$ \\
\hline $\begin{array}{l}\text { Senkung der } \\
\text { Stromsteuer }\end{array}$ & $\begin{array}{l}\text { „Die deutsche Wirtschaft braucht eine saubere, sichere } \\
\text { und bezahlbare Energieversorgung“ (S. 7). „Die EEG- } \\
\text { Umlage hat mittlerweile eine Höhe erreicht, die für private } \\
\text { Haushalte und weite Teile der Wirtschaft, insbesondere } \\
\text { auch mittelständische Unternehmen, zum Problem } \\
\text { wird,..."(S. 53) }\end{array}$ & SPD \\
\hline
\end{tabular}

Daneben gab es auch steuerpolitische Themen im Koalitionsvertrag, die in keinem der Wahlprogramme von CDU/CSU und SPD vorkamen, aber dennoch im Koalitionsvertrag auftauchten. Im Wahlkampf spielte die Frage der PKW-Maut, also einer Straßenbenutzungsgebühr, eine wichtige Rolle, da sich die Positionen von 
CDU und CSU hier deutlich unterschieden. So erklärte die Kanzlerin im Fernsehduell mit Peer Steinbrück explizit, dass die Maut nicht kommen würde. Bei der CSU gab es dagegen die Forderung nach einer Pkw-Maut für Autofahrer aus dem Ausland schon länger. Die SPD lehnte die Einführung einer Maut ab, obwohl sie diese früher gefordert hatte. Sowohl im Wahlprogramm der Union als auch der SPD waren keine Aussagen zur PKW-Maut zu finden. Der Koalitionskompromiss, ein Erfolg für die CSU, sah eine Regelung vor, bei der kein Fahrzeughalter in Deutschland stärker belastet wird als heute. Die Ausgestaltung soll, so auch der aktuelle Vorschlag des Verkehrsministers Dobrindt, EU-rechtskonform erfolgen.

Offensichtlich gelang es auch dem Bundesfinanzministerium, wichtige Themen im Koalitionsvertrag zu platzieren, obwohl diese im Vorfeld der Wahl weder in den Wahlprogrammen noch im Wahlkampf thematisiert wurden. Unter der Rubrik „Steuerhinterziehung bekämpfen - Steuervermeidung eindämmen“9 wurde eine Vielzahl von Maßnahmen gegen unlauteren Steuerwettbewerb und gegen Steuerhinterziehung thematisiert, die aktuell auch auf europäischer Ebene wichtig sind. Ziel ist zum einen die erhöhte Transparenz im internationalen Wettbewerb und zum anderen die Erfassung von im Ausland erzielten Einkünften. Zwar wurde in den beiden Wahlprogrammen von CDU/CSU sowie SPD angekündigt gegen Steuerbetrug und Steuerhinterziehung vorzugehen, doch war die Detailliertheit der Maßnahmen im Koalitionsvertrag im Vergleich zu den eher unverbindlichen Ankündigungen überraschend. $\mathrm{Zu}$ den wichtigsten Maßnahmen zählen dabei unter anderem folgende: Mit der sogenannten BEPS-Initiative (Base Erosion and Profit Shifting) sollen Lizenzaufwendungen, Transfer Pricing und Betriebsausgabenabzüge besser erfasst und kontrolliert werden. Außerdem sollen Maßnahmen gegen Umsatzsteuerbetrug ergriffen werden; vor allem soll das Bundeszentralamt für Steuern (BZSt) ausgebaut werden, um Steuerhinterziehung zu vermeiden. Auf europäischer Ebene wird eine weitere Harmonisierung der Unternehmensbesteuerung angestrebt. Die strafbefreiende Selbstanzeige soll ,weiterentwickelt werden“, was mittlerweile durch eine Gesetzesnovellierung auch auf dem Weg gebracht wurde.

Schließlich gibt es steuerpolitische Themen, die relevant sind, aber nicht im Koalitionsprogramm erwähnt werden. Nachdem 2012 das „Gesetz zum Abbau der kalten Progression“ am Widerstand der SPD-Bundesländer im Bundesrat gescheitert

9 Vgl. Koalitionsvertrag zwischen CDU, CSU und SPD, Deutschlands Zukunft gestalten, 18. Legislaturperiode, 2013, 90 . 
war, gelang es der CDU/CSU nicht diesen Punkt durchzusetzen bzw. im Koalitionsvertrag zu verankern. Die kalte Progression, die eine schleichende Steuererhöhung darstellt, da steigende Löhne stärker besteuert werden, wird daher wohl auch kaum in der aktuellen Legislaturperiode abgemildert werden, obwohl zwischenzeitlich von Teilen der SPD Gesprächsbereitschaft signalisiert wurde.

\section{Ausgabenentscheidungen der Bundesregierung}

Das Ausgabenwachstum ist für die kommenden Jahre moderat und die Ausgaben liegen aktuell für 2014 etwa auf dem Niveau wie für 2009. Auch für die kommenden Jahre sind im Finanzplan Ausgabensteigerungen unterhalb des Wirtschaftswachstums prognostiziert, was ein Fortschreiten auf dem Konsolidierungspfad bedeutet. Jedoch hat die Bundesregierung im ersten Jahr ihrer Amtszeit verschiedene Ausgabenentscheidungen getroffen, die zu einer Ausweitung der Staatstätigkeit Deutschlands führen werden. Vor dem Hintergrund der demographischen Entwicklung sind diese Ausgaben in ihrer langfristigen Wirkung fiskalisch noch nicht voll abzuschätzen. Hierzu gehören die zahlreichen Maßnahmen in der Rentenversicherung mit mehreren Leistungsverbesserungen. Von besonderer Tragweite waren dabei verschiedene Entscheidungen in der Rentenversicherung, wie

- die Einführung der Mütterrente,

- die Rente ab 63,

- Verbesserungen bei der Erwerbsminderungsrente,

- die solidarische Lebensleistungsrente.

Die Mütterrente war ein Projekt der Unionsparteien. Ab 1. Juli 2014 werden für alle Mütter oder Väter, deren Kinder vor 1992 geboren wurden, die Erziehungsleistung mit einem zusätzlichen Entgeltpunkt in der Alterssicherung berücksichtig. Dies entspricht einer Erhöhung der monatlichen Rente um 25,74 Euro in Ost- und 28,14 Euro in Westdeutschland je Kind. Insgesamt betragen die Kosten rund 6,5 Mrd. Euro jährlich.

Die Rente mit 63, ursprünglich ein Projekt der SPD, ermöglicht langjährig Versicherten, die 45 Beitragsjahre (einschließlich Zeiten der Arbeitslosigkeit) in der Rentenversicherung aufweisen, ab dem 1. Juli 2014 mit dem vollendeten 63. Lebensjahr abschlagsfrei in Rente gehen. Allerdings wird das Zugangsalter, mit dem der abschlagsfreie Rentenzugang möglich ist, schrittweise parallel zur Anhebung des allgemeinen Renteneintrittsalters auf das vollendete 65. Lebensjahr angehoben. Schätzungen gehen von etwa 5 Mrd. Euro Kosten pro Jahr aus. 
Die Verbesserungen bei der Erwerbsminderungsrente, ebenfalls ein Projekt der SPD, beinhalten eine Anhebung der Zurechnungszeit bei der Erwerbsminderungsrente zum 1. Juli 2014 um zwei Jahre (von 60 auf 62). Die Kosten sollen rund 800 Millionen Euro p.a. betragen.

Die CDU hat sich mit der Einführung der solidarischen Lebensleistungsrente für Geringverdiener, die voraussichtlich 2017 eingeführt wird, durchgesetzt. Wer mindestens 40 Jahre in der gesetzlichen Rentenversicherung versichert war und nur eine geringe Rente erreicht (weniger als 30 Rentenentgeltpunkte), wird durch eine Aufwertung bessergestellt werden.

Diese Maßnahmen werden aus unterschiedlichen Quellen finanziert: So wurde bereits zwei Tage nach Vereidigung der neuen Bundesregierung ein Stopp der Beitragssenkung in der Rentenversicherung im Bundestag verabschiedet. Die eigentlich gesetzlich notwendige Senkung des Beitragssatzes von 18,9 \% auf 18,3\% wurde dadurch verhindert. Arbeitgeber und Arbeitnehmer müssen damit auf eine Entlastung von ca. sechs Milliarden Euro pro Jahr verzichten. Zudem werden Haushaltsüberschüsse, die zur Schuldentilgung verwendet hätten werden können, hierfür eingesetzt. Außerdem wird von einem anhaltend tiefem Zinsniveau ausgegangen, um die ausgestellten „Rentenschecks“ zukünftig zu finanzieren.

Wie stellen sich diese Umbrüche und Herausforderungen auf den unterschiedlichen einzelnen staatlichen Ebenen dar? Dies sind die zentralen Leitfragen des vorliegenden Doppelheftes der ZSE. Ist die Situation des öffentlichen Gesamthaushaltes auf allen staatlichen Ebenen positiv oder stellt sich im Hinblick auf einzelne Länder oder bestimmte Kommunen die Situation doch nicht sehr viel differenzierter dar, wie es die sehr hoch aggregierten Zahlen erscheinen lassen. Einen näheren Blick auf den Zustand der öffentlichen Finanzen zu werfen, ist dabei ein Hauptziel dieses Sonderheftes. Dabei werden sowohl die europäische Ebene, die Nationalstaaten sowie die Länder und Kommunen in den Blick genommen. Dies geschieht überdies aus mehreren Perspektiven: Einerseits aus der Perspektive von Politikern und mit Entscheidungen befassten Akteuren und andererseits aus der Perspektive von Wissenschaftlern. Dieser Mix wird zudem ergänzt durch eine Mischung der Wissenschaftsdisziplinen aus denen die Autoren des Heftes kommen. So sind Juristen, Ökonomen, Soziologen sowie Politikwissenschaftler die Autoren dieses Doppelheftes. Schließlich wurde nicht nur bei den Politikern auf unterschiedliche Parteifärbung Wert gelegt, sondern es finden sich sowohl befürwortende als auch kritische Positionen gegenüber einer Haushaltskonsolidierung. 
Aus der Sicht der Bundesregierung stellt der Beitrag von Staatssekretär Steffen Kampeter die Vorhaben im Bereich der Steuerpolitik und der Staatsausgaben sowie der Hauptpriorität der Bekämpfung der Defizite in den Mittelpunkt. Neben der Fokussierung auf die Fiskalregeln liegt ein wichtiger Schwerpunkt auf einer weiteren institutionellen Vorkehrung gegen die Verschuldung: Die Änderung des Haushaltsvollzugs und der Haushaltsplanung. Staatssekretär Kampeter macht dabei deutlich, dass allein die Fiskalregeln wohl nicht ausreichen werden, um die Verschuldung nachhaltig zu begrenzen. Komplementär zur Schuldenregel sind die bereits in anderen Ländern schon länger eingeführten Top-Down-Elemente einer Haushaltsaufstellung wichtig. Diese Verfahren spielen im Übrigen auch in der Analyse von Jobst Fiedler und Quirin Maderspacher eine zentrale Rolle, die die Länderhaushalte vergleichend untersuchen. Der Bundeshaushalt, mittlerweile zum 4. Mal nach dem Top-Down-Verfahren aufgestellt, hat als wesentliches Element Eckwertebeschlüsse, die vom Kabinett beschlossen werden und dann in der Haushaltsaufstellung - und natürlich auch im Vollzug - einzuhalten sind. Die hohe Bindungskraft dieser Regeln wird in der Literatur zur Haushaltskonsolidierung besonders hervorgestellt, ist jedoch in der Öffentlichkeit bisher noch nicht wahrgenommen worden. Staatssekretär Kampeter verweiset daneben noch auf die internationale Ebene mit dem gestärkten Stabilitäts- und Wachstumspakt, der durch verschiedene Instrumente (u.a. „Sixpack“, Einführung des europäischen Semesters) eine Intensivierung der haushaltspolitischen Überwachung vorsieht. In wieweit jedoch diese Maßnahmen auf europäischer Ebene Erfolg zeigen, bleibt abzuwarten. Daneben geht Staatssekretär Kampeter auch auf die Entwicklung der Finanzbeziehungen nach 2019 ein, wobei das Ziel der Bundesregierung ist, ein langfristig tragfähiges System zu erhalten, welches weniger in die öffentliche Auseinandersetzung gerät als in der Vergangenheit.

Der Präsident des Deutschen Städtetages, Ulrich Maly, fokussiert sich in seinem Beitrag auf die kommunale Ebene. Ausgehend vom normativen Leitbild des Selbstverständnisses der kommunalen Ebene, die den Städten vor allem auch eine eigene Gestaltungskompetenz zuschreibt und nicht nur bloßes Verwaltungshandeln bzw. Verwaltungsvollzug zubilligt, stellt der Städtetagspräsident die Beibehaltung der Handlungs- und der Gestaltungsfähigkeit der kommunalen Ebene ins Zentrum seiner Ausführungen. Im Hinblick auf die öffentlichen Finanzen konzediert Maly, der gleichzeitig der Oberbürgermeister Nürnbergs ist, zwar eine gewisse Verbesserung im Aggregat, bemerkt aber, dass insbesondere die Kassenkredite mittlerweile zu einem dauerhaften Finanzierungsinstrument der laufenden Ausgaben geworden sind. Zudem gibt es einen Teufelskreislauf zwischen hohen 
Sozialausgaben, abnehmenden Investitionen und einer nachlassenden Standortattraktivität, während auf der anderen Seite steigende Steuersätze und damit eine insgesamt nachlassende Wettbewerbsfähigkeit von Kommunen resultiert. Städtetagspräsident Maly identifiziert dabei eine große regionale Varianz zwischen einzelnen Gebieten, ein Befund, der auch in der detaillierten Analyse der kommunalen Kassenkredite in dem Beitrag von Martin Junkernheinrich und Uwe Wagschal empirisch bestätigt wird. Ein großes Problem der kommunalen Finanzen ist dabei der Rückgang der Investitionstätigkeit. Daneben macht Maly auch eine gewisse Skepsis gegenüber Fiskalregeln deutlich. Insbesondere wenn solche Regeln zu einer Entmündigung der kommunalen Selbstverwaltung führen. Die Erwartung, die die Kommunen an den Bund und die Länder formulieren, ist vor allen Dingen diejenige, dass die Konsolidierungsanstrengungen nicht auf die Kommunen abgeladen werden.

Der Staatsrechtslehrer Werner Heun aus Göttingen richtet in seinem Beitrag den Blick vor allem auf die Verfassungsrechtsprechung und ihr komplexes Verhältnis zu den öffentlichen Finanzen. Heun stellt dabei fest, dass einige Bereiche der öffentlichen Finanzen, wie etwa die Finanzausgleichsregelung und die Staatsverschuldung, vergleichsweise spät in den Fokus des Verfassungsgerichts gekommen sind. Steuerrechtliche Entscheidungen wurden dagegen sehr viel häufiger und früher vom Verfassungsgericht beurteilt. Insgesamt kommt er dabei zu einem ambivalenten Ergebnis, wenn man die Rechtsprechung des Verfassungsgerichts Revue passieren lässt. Im Bereich des Haushalts- und Finanzverfassungsrechts hat es wirkungsvolle Anstöße gegeben, während beim Finanzausgleich das Verfassungsgericht oftmals an Grenzen der Steuerungsfähigkeit gelangt. Im Bereich der Staatsverschuldung ist überdies die Einflussmöglichkeit oftmals gering, da die Entscheidung teilweise ignoriert wird und mitunter erst auch mit großer Verspätung Entscheidungen getroffen wurden.

Lukas Haffert und Philip Mehrtens vom Max-Planck-Institut für Gesellschaftsforschung in Köln gehen in ihrer Untersuchung der Frage nach, ob die Haushaltsüberschüsse nach Konsolidierungserfolgen tatsächlich eine größere staatliche Handlungsfähigkeit ermöglichen. Insbesondere schauen sie sich die unterschiedlichen Ausgabenprofile der einzelnen Länder an, die Überschüsse erzielt haben. Bemerkenswert ist, dass zahlreiche OECD Länder zumindest phasenweise Überschüsse erzielt haben (Neuseeland z.B. 15 Jahre). Es zeigt sich in der Analyse, dass vor allen Dingen die Investitionen zurückgefahren wurden und generell diese einem abnehmenden Trend unterworfen sind. Vor dem Hintergrund möglicher Haushaltsüberschüsse zeigen die Befunde des internationalen Vergleiches, dass 
offensichtlich vorhandene Handlungsspielräume nicht genutzt worden sind, um höhere Investitionen zu tätigen. Eine gewisse Skepsis für sich möglicherweise dauerhaft abzeichnender Haushaltsüberschüsse in Deutschland ist damit eines der wichtigen Ergebnisse von Haffert und Mehrtens.

Der Beitrag von Klaus Armingeon, Kai Guthmann und David Weisstanner beschäftigt sich ebenfalls mit dem internationalen Vergleich von Austeritätspolitiken. Vor dem Hintergrund eines theoretischen Ansatzes, der (1) auf der Parteiendifferenztheorie, (2) der Theorie der Machtressourcen von organisierten Interessen sowie (3) auf den institutionellen Gegebenheiten eines Landes (Vetospielertheorie) basiert, analysieren sie die Haushaltskonsolidierung in 17 OECD Ländern. Insbesondere wird unterstellt, dass bürgerliche Parteien eher fiskalisch konsolidieren als linke Parteien. Bemerkenswerterweise zeigen die Befunde, dass rechte Regierungen weniger konsolidieren als Regierungen anderer Parteifärbungen. Außerdem ist die Größe der Regierungskoalitionen von Bedeutung: Größere Regierungen führen umfangreichere Konsolidierungen durch als kleinere Regierungen. Insofern stellen die Autoren für den internationalen Vergleich sowohl Parteieneffekte als auch institutionelle Effekte fest. Kleinere Koalitionsregierungen konsolidieren dafür häufiger bei geringerem Umfang.

Nach dem internationalen Vergleich lenken Jobst Fiedler und Quirin Maderspacher von der Hertie School of Governance in Berlin den Blick auf die Wirkungen der Fiscal Governance in deutschen Bundesländern. Bereits im Heft 4/2013 der ZSE haben Ciaglia und Heinemann ${ }^{10}$ einen neuen Index zur Erfassung der Rigidität der Verfassungsgrenzen auf Bundesländerebene eingeführt. Der hier nun vorgelegte Index von Fiedler und Maderspacher greift diesen Ansatz auf, geht jedoch noch darüber hinaus, indem er mehr Indikatoren und auch die tatsächliche Wirkung der Regelungen versucht empirisch zu erfassen. Damit stehen sie in der Tradition von vergleichenden Indikatoren der Fiscal Governance auf Bundesländerebene. ${ }^{11}$ Der so entwickelte Index wird anschließend empirisch getestet, indem die Wirkung auf die verschiedenen Verschuldungsindikatoren untersucht wird. Auch hier zeigt sich, dass Top-Down-Instrumente, wie bereits im Beitrag von Staatssekretär Kampeter betont, einen wichtigen Einfluss auf die Sparanstrengungen besitzen. Institutionelle Selbstbindung durch stärkere Haushaltsregeln, aber auch die Ausrichtung des Budget-Prozesses auf Top-Down-Verfahren, bringen -

10 Vgl. Ciaglia, S./ Heinemann, F.: Debt Rule Federalism: The Case of Germany, ZSE: Zeschrift für Staats und Europawissenschaften, 4/2013, 570-602.

11 Vgl. Wagschal, U./ Wintermann, O./ \& Thieß, P.: Konsolidierungsstrategien der Bundesländer, Gütersloh, 2008. 
so die Autoren - insgesamt bessere Haushaltsergebnisse hervor. Damit bestätigen die Autoren überdies Vergleichsergebnisse aus dem internationalen Vergleich.

Noch eine staatliche Ebene tiefer gehen Martin Junkernheinrich und Uwe Wagschal in ihrer empirischen Analyse der Kassenkredite als Teil der kommunalen Verschuldung. Dabei untersuchen sie in einem quantitativen Vergleich die Haushaltsdaten der bundesdeutschen Kommunen und identifizieren so die zentralen Ursachen, insbesondere sozioökonomische Faktoren, für die Kommunalverschuldung. Neben den Determinanten dieser Kommunalverschuldung diskutieren die Autoren überdies noch die möglichen Lösungen für die Beseitigung exzessiver Kassenkredite.

Der Blick auf die europäische Ebene richten Matthias Mors und Stefan Appel von der EU Kommission. Die Autoren analysieren am Beispiel Griechenlands die Politik der Troika, das heißt der Europäischen Kommission der Europäischen Zentralbank und des Internationalen Währungsfonds bei der Ausgestaltung der ökonomischen Anpassungsprogramme für die Mitgliedstaaten der Eurozone. Gerade das Beispiel Griechenlands stand besonders im Mittelpunkt der politischen Auseinandersetzungen der vergangenen Jahre. Mit dem Schuldenschnitt im Jahre 2012 wurden dort auch drastische Maßnahmen im Zuge der Hilfsprogramme durchgeführt. Der Insiderbericht zur Politik der Troika lässt zum ersten Mal einen intensiven Blick in die Arbeit dieser neuen ad hoc gebildeten Institution zu und ermöglicht einen realistischen Blick auf die Arbeit der Troika.

Ingolf Deubel, ehemaliger Finanzminister des Landes Rheinland-Pfalz, veröffentlicht in seinem Beitrag eine neue und innovative Herangehensweise zur Analyse der Bundesländer-Finanzen. Vor dem Hintergrund der Schuldenbremse und der Neuordnung der Länderfinanzen 2020 analysiert Deubel zunächst einmal die unterschiedliche Finanzausstattung der Länder im Pro-Kopf-Vergleich und im relativen Vergleich. In einem stufenweisen Schritt schichtet Deubel anschließend Zinsen, Versorgung, Sozialausgaben und Sachinvestitionen von den Bruttoausgaben ab und kommt am Ende zu einem empirischen Vergleich, der die faktische Finanzmöglichkeit der Länder angeht. Im zweiten Schritt führt Deubel dann Simulationsrechnungen durch und schätzt den zur Verfügung stehenden Finanzrahmen für das Jahr 2020 ab. Insbesondere wird dabei von unterschiedlichen Zinsniveaus und Steuerentwicklungen ausgegangen. Insgesamt stellen sich auf Basis dieses Ländervergleichs eindrucksvoll die möglichen Handlungsspielräume und Konsolidierungsbedarfe dar, um ab 2020 die Schuldenbremse einhalten zu können. 